\title{
Trauma registries in Europe
}

\author{
R. Lefering $\cdot S$. Ruchholtz
}

Received: 22 November 2011/Accepted: 23 November 2011/Published online: 16 December 2011

(C) Springer-Verlag 2011

The management and therapy of severe trauma is an important task in medicine, as trauma victims are mostly young people, and the mortality rate for severe trauma is still high. The treatment of trauma patients is cost-intensive, with an average intensive care unit stay of two weeks for survivors (ISS $\geq 16$ ), and sometimes an extended phase of rehabilitation. Furthermore, many resources are needed to keep an emergency medical system ready on a 24 h/7 days a week basis, including ambulance cars, helicopters, and trained personnel. Trauma centers that are properly prepared to treat severely injured patients require several structural features, including intensive care units, trauma units, diagnostic devices such as computer tomographs and a blood bank, as well as a trained team incorporating different disciplines (orthopedic and visceral surgeons, anesthesiologists, radiologists, neurosurgeons, nurses, etc.).

Despite the importance of this for the patient as well as for society, there is only limited evidence for most of the diagnostic and therapeutic strategies that are applied to severely injured patients. Classical randomized trials-the best method of producing scientific evidence-are often very difficult or even impossible to perform in the setting of severe trauma care. There are many reasons for this. Patients are usually unable to provide informed consent, the time to react in a given situation is often very limited, and a large number of professionals are involved in the

\section{R. Lefering $(\bowtie)$}

IFOM, Institute for Research in Operative Medicine,

University of Witten/Herdecke, Cologne, Germany

e-mail: rolf.lefering@uni-wh.de

\section{S. Ruchholtz}

Department of Trauma Surgery, University of Marburg,

Marburg, Germany treatment chain from prehospital care until discharge. Therefore, other sources of scientific information, such as registries, are of high importance.

Trauma registries are often found in individual trauma centers where performance and management decisions are documented internally. However, the great value of trauma registries lies in the potential to compare institutions-i.e., to compare different institutions, or to compare the same institution at different times. This of course requires structured, consistent data collection with uniform definitions. Regional and national trauma registries have created such a system, and even at the international level such activities are underway. Trauma registries in Europe have reached a consensus agreement on a core dataset for future comparative analyses [1]. A validation study just appeared in the journal Critical Care [2]. This is the first step in the direction of a joint European trauma registry.

However, results from trauma registries must also be interpreted with caution. Unlike in randomized trials, the comparability of subgroups in registries cannot generally be taken for granted (this refers to comparisons between different hospitals, different diagnostic or therapeutic strategies, or time periods). Sophisticated statistical methods are needed to minimize bias and to reach a level of comparability that allows at least some major effects or trends to be identified. Such methods include subgroup analyses, parallelization, propensity score analyses, matched pair analyses, and outcome adjustment with prognostic scores. The big advantage of registries is, however, that there is hardly any limitation on sample size.

Examples from existing trauma registries show that registry-based analyses are worth publishing, even in the highest-ranked medical journals [3, 4].

Finally, the quality of the results depend crucially on the quality of the data. Therefore, measures to ensure and 
increase the quality of data in trauma registries are mandatory. In this regard, it should be stated that the quality of registry data can be described in three dimensions. The first dimension refers to the completeness regarding the number of cases (i.e., are all, or at least most, of the appropriate cases actually included in the registry?). If completeness cannot not be attained, the data should at least be representative. The second dimension refers to the completeness of the data for each case documented in the registry (are all data available for each case, or are there parameters with a substantial amount of missing data?). The third and final dimension is the correctness of the data, which can be improved by performing plausibility checks and sample source data verification.

The present issue of the European Journal of Trauma and Emergency Surgery focuses on trauma registries, and gives some examples of scientific analyses based on registry data. Such research enables trauma registries to constantly contribute to the body of scientific evidence in trauma care-and this research is of course of enormous value for interhospital comparisons and quality assurance. For example, the Trauma Registry of the German Society for Trauma Surgery (DGU) repeatedly showed that quality of care is very heterogeneous among participating hospitals. How can this be improved?

Data from the last few decades show that regionalizing trauma care can be highly effective for optimizing the treatment of severely injured patients, and that this approach can reduce mortality [5]. Consequently, in 2006, the DGU established the TraumaNetwork DGU ${ }^{\circledR}$ project. The aim of this project was to optimize the treatment of severely injured patients by standardizing processes, structures, education, and personnel in all trauma centers that are involved in the treatment of these patients. In the current issue, an article from the steering group of the TraumaNetwork DGU ${ }^{\circledR}$ project describes the implementation of these quality-oriented networks. Trauma registry data will also help to verify the advantages for patients.

Conflict of interest None.

\section{References}

1. Ringdahl KG, Coats TJ, Lefering R, di Bartolomeo S, Steen PA, Røise O, Handolin L, Lossius HM, and Utstein TCD Expert Panel. The Utstein template for uniform reporting of data following major trauma: a joint revision by SCANTEM, TARN, DGU-TR and RITG. Scand J Trauma Resusc Emerg Med. 2008;16:7.

2. Ringdal KG, Lossius HM, Jones JM, Lauritsen JM, Coats TJ, Palmer CS, Lefering R, Di Bartolomeo S, Dries DJ, Søreide K and The Utstein Trauma Data Collaborators. Collecting core data in severely injured patients using a consensus trauma template: an international multicentre study. Critical Care 2011;15:R237.

3. Huber-Wagner S, Lefering R, Qvick LM, Körner M, Kay MV, Pfeifer KJ, Reiser M, Mutschler W, Kanz KG, and the Working Group on Polytrauma (NIS) of the German Trauma Society (DGU). Whole body computed tomography during trauma resuscitation-effect on outcome. Lancet. 2009;373(9673):1455-61.

4. Patel HC, Bouamra O, Woodford M, King AT, Yates DW, Lecky FE (TARN). Trends in head injury outcome from 1989 to 2003 and the impact of neurosurgical care: an observational study. Lancet. 2005;366:1538-44.

5. MacKenzie EJ, Rivara FP, Jurkovich GJ et al. A national evaluation of the effect of trauma-center care on mortality. N Engl J Med. 2006;354(4):366-78. 